\title{
Marching towards making evolution-proof antibiotics
}

\begin{abstract}
Antibiotic resistance in bacterial pathogens has increased to alarming levels and warrants immediate attention. Traditional antibiotics kill the bacteria by targeting the bacterial structures and mechanisms that are essential for their survival, putting great selection pressure to develop resistance. Anti-virulence drugs are new class of therapeutic targets being investigated to tackle this crisis. As anti-virulence drugs disarm the pathogen rather than killing, it may pose less selection pressure on bacteria to develop resistance.
\end{abstract}

Keywords: antibiotics, anti-virulence drugs, penicillin, rheumatoid arthritis, T3SS
Volume 5 Issue 5 - 2017

\author{
Veerendra Koppolu \\ Department of Molecular Biosciences, University of Kansas, \\ USA \\ Correspondence: Veerendra Koppolu, Department of \\ Molecular Biosciences, University of Kansas, Lawrence, KS, USA, \\ Tel+| 67869792|6, Email veeru.bios@gmail.com
}

Received: October 30, 2017 | Published: November 03, 2017

\section{Introduction}

Discovery of the first antibiotic in 1928 by Alexander Fleming is considered as the greatest advance in therapeutic medicine and surgery. Penicillin was highly successful in controlling bacterial infections during World War II. Since then, many antibiotics were discovered to treat multiple bacterial infections and these antibiotics undoubtedly saved millions of lives. Antibiotics successfully prevented or treated hospital-acquired bacterial infections that can occur in patients during complex surgeries such as organ transplantations, joint replacements or cardiac surgery; patients receiving chemotherapy; and patients suffering from chronic diseases such as diabetes, renal diseases, and rheumatoid arthritis etc.

The efficacy of antibiotics in treating bacterial infections is rapidly endangering due to the emergence of bacterial resistance to antibiotics. Resistance has been evident against all the antibiotics that were discovered. A growing number of infections such as tuberculosis, pneumonia, gonorrhea, and some diarrheal infections are becoming harder to treat or sometimes impossible as antibiotics against them have become less effective. In the USA alone, hospital-acquired infections account for an annual 2 million new case and 99000 deaths, mostly due to resistant bacterial pathogens. ${ }^{1}$ According to world health organization (WHO) estimates, drug-resistant tuberculosis itself accounts for more than 480000 new cases. ${ }^{2}$ The rapid emergence of resistance calls for urgency in understanding the reasons for resistance and ways to mitigate the resistance development.

The crisis aroused due to antibiotic resistance is mainly attributed to, current choice of antibiotic target mechanisms, overuse and misuse of antibiotics, and lack of new drug development by pharmaceutical industry. Antibiotics developed so far target the mechanisms essential for bacterial survival such as protein synthesis, cell wall formation, and growth. Targeting these mechanisms put extensive pressure on the bacteria to develop resistance. The misuse and overuse of these antibiotics further aggravate the resistance bacterial strain development. The development of new antibiotics by pharmaceutical industry, an option used to combat drug resistance by bacteria in the past is slowed down as more and more companies are not considering antibiotic discovery as an economically viable option. While there are some new antibiotics under development, none of them are expected to treat multi drug-resistant bacteria. In recent years, the development of new antibiotics has generally been limited to modifications of existing antibiotics, which again primarily target essential bacterial proteins and put extensive selective evolutionary pressure on bacteria to develop resistance. In addition, current broad-spectrum antibiotics adversely affect the resident gut microbiota. These events eventually led to Center for Disease control (CDC) declaration in 2013 that humanity is now in "Post-antibiotic era" and WHO warning in 2014 that the antibiotic resistance crisis is dire.

Keeping the possible antibiotic resistance in mind, it is of greatest need to develop new ways to treat bacterial infections. Researchers are marching in this direction to make evolution-proof antibiotics by targeting the ways that will disarm the bacterial pathogens without impacting their survival. Disarming the pathogen rather than killing or halting its growth is considered as an important strategy towards sidestepping the evolution of resistance. Drugs that disarm the pathogen are hypothesized to generate much weaker selection for resistance than traditional antibiotics. Disarming the pathogens is possible by targeting the virulence mechanisms which are crucial for bacterial pathogenicity. Researchers have been targeting a conserved family of bacterial proteins called AraC family proteins that are present in $\sim 70 \%$ of bacteria and are known to regulate bacterial virulence, stress response, and sugar metabolism. By targeting these AraC family regulatory proteins, bacteria can be made non-pathogenic and the surveilling immune cells can identify the non-infective bacteria and clear them from the body. This exciting hypothesis has been tested in Shigella flexneri, a diarrhea-causing bacteria pathogen responsible for causing 165 million cases of illness and more than 1.1 million deaths worldwide each year, and $70 \%$ of those dying from illnesses due to Shigella are children under the age of 5. A group of researchers lead by Dr. Susan Egan selectively inhibited an important AraC family Shigella virulence regulatory protein VirF that regulates a type III secretion system (T3SS) required for bacterial invasion. ${ }^{3}$ A highly potent molecule "SE-1" ( 1-butyl-4-nitromethyl-3-quinolin2-yl-4H-quinoline) is found to not impact the growth of the bacteria but prevent bacteria's ability to invade and infect cultured human intestinal cells. The efficacy and solubility of the molecule SE-1 is high enough to be developed as novel-antibacterial agent. ${ }^{46}$ SE-1 also inhibits infection pathways in other pathogenic bacteria that cause infections such as diarrhea, pneumonia, and cholera and thus can be developed as a novel agent to treat multiple infections.

In addition to the AraC family regulators, components of type III secretion system (T3SS) including the needle tip complex and T3SS associated cytosolic ATPases have been considered as promising antivirulence targeting choices. ${ }^{7-11}$ Considering the conservation of T3SS across many pathogenic bacteria, discovery of an effective T3SS virulence blocker may yield non-traditional antibiotics that are more 
powerful and versatile than our current antimicrobials. Because the bacteria will still be able to survive in the presence of these drugs, there should be no longer a selection pressure on the bacteria to evolve resistance.

\section{Acknowledgements}

None.

\section{Conflict of interest}

The author declares no conflict of interest.

\section{References}

1. Ventola CL. The antibiotic resistance crisis: part 1: causes and threats. PT. 2015;40(4):277-283.

2. World health Organization. Antimicrobial resistance. 2016.

3. Koppolu V, Osaka I, Skredenske JM, et al. Small-Molecule Inhibitor of the Shigella flexneri Master Virulence Regulator VirF. Infect Immun. 2013;81(11):4220-4231.

4. Skredenske JM, Koppolu V, Kolin A, et al. Identification of a SmallMolecule Inhibitor of Bacterial AraC Family Activators. $J$ Biomol Screen. 2013;18(5):588-598.
5. Koppolu V. Molecular Mechanisms and Inhibition of Transcription Activation by Bacterial AraC Family Activator Proteins. USA: University of Kansas; 2013.

6. Jain P, Li J, Porubsky P, et al. 3-Substituted Biquinolinium Inhibitors of AraC Family Transcriptional Activator VirF from S. flexneri Obtained Through In Situ Chemical Ionization of 3,4-Disubstituted Dihydroquinolines. RSC Adv. 2014;4(75):39809-39816.

7. Muschiol S, Bailey L, Gylfe A, et al. A small-molecule inhibitor of type III secretion inhibits different stages of the infectious cycle of Chlamydia trachomatis. Proc Natl Acad Sci USA. 2006;103(39):14566-14571.

8. Wolf K, Betts HJ, Chellas-Gery B, et al. Treatment of Chlamydia trachomatis with a small molecule inhibitor of the Yersinia type III secretion system disrupts progression of the chlamydial developmental cycle. Mol Microbiol. 2006;61(6):1543-1555.

9. Negrea A, Bjur E, Ygberg SE, et al. Salicylidene acylhydrazides that affect type III protein secretion in Salmonella enterica serovar typhimurium. Antimicrob Agents Chemother. 2007;51(8):2867-2876.

10. Veenendaal AK, Sundin C, Blocker AJ. Small-molecule type III secretion system inhibitors block assembly of the Shigella type III secreton. $J$ Bacteriol. 2009;191(2):563-570.

11. Valasani KR, Kuruva CS, Koppolu V, et al. Synthetic and Biological Applications of Benzothiazole Phosphonates. Heterocyclic Compounds and Biological Applications. USA: Science Publishing Group; 2017. 